\title{
आरण्यक ग्रन्थों में संगीत
}

प्रो. पंकज माला शर्मा

संगीत विभाग, पंजाब विश्वविद्यालय, चण्डीगढ़

भारतीय संगीत के इतिहास का प्रसंग आते ही वैदिक साहित्य की ओर ध्यान केन्द्रित हो जाता है। वेद जो सम्पूर्ण ज्ञान विज्ञान का आधार है वही संगीत का भी आधार है। साक्षात्कृत धर्मा ऋषियों के अन्त: करण में तपस्या द्वारा जो ज्ञान उद्भूत हुआ उसकी शब्दात्मक अभिव्यक्ति ऋक्, यजुष, तथा साम के रूप में हुई। यही वेद का मन्त्रात्मक स्वरूप था जो कि पद्य, गद्य तथा गान के रूप में था। एक समय ऐसा आया जब मन्त्ररचना की प्रवृति समाप्त हो गयी। ऋषि परम्परा में इन सृष्ट मन्त्रों के संरक्षण के लिए उनके मौखिक संकलन तैयार किये गये। सभी वंश के ऋषियों के मन्त्रों को एकत्र करके योजनाबद्ध संकलित करने का कार्य कृष्णद्वैपायन व्यास के द्वारा किया गया। उनके द्वारा संकलित चार संहितायें वेद के नाम पर प्रचलित हुई जिन्हें ऋग्वेद, यजुर्वेद, सामवेद तथा अथर्ववेद के नाम से जाना जाता ह। अध्ययन-अध्यापन की परम्परा में मन्त्रों के क्रम तथा उच्चारण में भेद दृष्टिगोचर होने लगे। परिणामतः प्रत्येक वेद की अनेक शाखायें प्रचलित हो गयीं तथा शाखा संहितायें निर्मित हो गयीं। संगीत का सम्बन्ध सामवेद से है परन्तु ऋग्वेदादि अन्य संहिताओं में संगीत से सम्बन्धित संदर्भ प्राप्त होते हैं। जिस समय शाखा संहितायें सत्ता में आयों उसी समय उन संहिताओं के मन्त्रों का व्याख्यान करने वाले एक विशिष्ट प्रकार के साहित्य का सृजन हुआ जिन्हें 'ब्राह्मण' संज्ञा प्रदान की गयी। 'ब्रह्मन्' शब्द का प्रयोग मन्त्र के लिए किया गया है। इसलिए इन व्याख्यान ग्रन्थों को ब्राह्मण कहा गया। 'बह्म' यज्ञ को भी कहते हैं। इसलिए यज्ञ विज्ञान का विवेचन करने के कारण भी इन्हें ब्राह्मण कहा जाता है। ब्रह्म से ही सृष्टि विकसित हुई। इसलिये यज्ञ विज्ञान के साथ सृष्टि विद्या का प्रतिपादन भी इन ब्राह्मण ग्रन्थों में प्राप्त होता है। जितनी शाखासंहितायें थी उतने ही उनके व्याख्यान रूप ब्राह्मण ग्रन्थ भी रहे होंगे ऐसा अनुमान किया जा सकता है किन्तु आज संहिताओं की भांति उपलब्ध ब्राह्मणों की संख्या बहुत कम है। आज प्राप्त होने वाले सम्पूर्ण ब्राह्मणों की संख्या 16 है। जिनमें सामवेद के आठ ब्राह्मण हैं। ब्राह्मणों के पश्चात् उसी का परिशिष्ट रूप एक विशिष्ट साहित्य जो वैदिक वाड्.मय के अन्तर्गत ही माना जाता है। 'आरण्यक' कहलाता है। आरण्यक साहित्य एवं उसमें निहित संगीत की जानकारी का उल्लेख स्वतन्त्र एवं स्पष्ट रूप से प्राप्त नहीं होता। प्रस्तुत शोध पत्र में आरण्यक का अर्थ, आरण्य साहित्य की विशेषता तथा उसका प्रतिपाद्य विषय, आरण्यकों की भाषा तथा उनमें निहित संगीत विषयक चिन्तन का अध्ययन करने का प्रयास किया गया है।

आरण्यक नाम से स्पष्ट है कि इन ग्रन्थों का घनिष्ठ सम्बन्ध 'अरण्य' से है। इन ग्रन्थों को आरण्य इसलिये कहा जाता है क्योकि इन ग्रन्थों का मनन अरण्य अर्थात वन में किया जाता था। ब्राह्मण ग्रन्थों के जो भाग 'अरण्य' में पठनीय हैं उन्हें 'आरण्यक' कहा जाता है। अत: यह स्पष्ट है कि आरण्यक का सम्बन्ध ब्राह्मण ग्रन्थों से है। कर्मकाण्ड के साथ दोनों का ही सम्बन्ध है इसलिये ये एक ही परम्परा से स्यूत है। 
आरण्यकों का देश काल वही है जो ब्राह्मण ग्रन्थों का है। तैत्तिरीय आरण्यक में गंगा-यमुना का तटवर्ती मध्यवर्ती मध्यदेश अत्यन्त पवित्र तथा मुनियों का निवास बताया गया है। इसी आरण्यक में आगे कुरुक्षेत्र तथा खाण्डव वन का वर्णन है जिससे ज्ञात होता है कि इसका सम्बन्ध कुरु, पांचाल जनपदों से रहा है। ${ }^{2}$ शांखायन आरण्यक में उशीनाट मत्स्य, कुरु, पांचाल, काशी तथा विदेह जनपदों का वर्णन है। ${ }^{3}$ मैत्रायणी आरण्यक में तत्कालीन भारत के अनेक प्रतापी सम्राटों के नाम प्राप्त होते हैं। ${ }^{4}$ इससे यह स्पष्ट है कि किन्हीं आरण्यकों का समय महाभारत काल के समकक्ष है।

आरण्यकों की भाषा सामान्यत: ब्राह्मणग्रन्थों के सदृश ही है। यह वैदिक तथा लौकिक संस्कृत के मध्य की भाषा है। जैमिनी शाखा के तलवकार आरण्यक की भाषा में अन्य आरण्यकों की अपेक्षा, अधिक प्राचीन रूप सुरक्षित है। शैली वर्णनात्मक है तथा कहीं-कहीं संवादमूलक भी है।

जब हम आरण्यकों के प्रतिपाद्य विषय की बात करते हैं तो यह ध्यान में अवश्य रहता है कि आरण्य ब्राह्मणों का परिशिष्ट रूप है। ब्राह्मण ग्रन्थ यद्यपि सृष्टिविज्ञान यज्ञविज्ञान का विवेचन विशदरूप से करते हैं। किन्तु उनमें वैद्ययजों के विवेचन में उनका यह दृष्टिकोण प्रमुरव रहा है। कि उन यजों का विधिपूर्वक सम्पादन भी किया जा सके। किन्तु इसके विपरीत आरण्यकों में यज्ञों के कर्मकाण्डीय पक्ष को प्रधानता न देकर उनके आध्यात्मिक विवेचन तथा इन्द्रिय, वाक्, मन, प्राण आदि विषयों के विवेचन को अधिक महत्त्व दिया गया है। इन विषयो का चिन्तन अरण्य में किया जाता था इसलिये इनकी 'आरण्य' संज्ञा थी। रहस्यात्मक गूढ़ विषयों का विवेचन करने के कारण ये 'रहस्य' नाम से भी प्रसिद्ध थे।

यद्यपि आरण्यक मूलतः ब्राह्मणों के ही भाग थे किन्तु आज ये अलग से भी प्रकाशित है। उपलब्ध आरण्यकों में से ऐतरेय आरण्यक तथा शांखायन आरण्यक ऋग्वेद के ब्राह्मणों से सम्बद्ध हैं। माध्यन्दिन बृहदारण्यक तथा काण्व बृहदारण्यक शुक्ल यजुर्वेद के ब्राह्मणों से सम्बद्ध हैं। कृष्ण यजुर्वेद के तीन आरण्यक है- तैतिरीय आरण्यक, काठक आरण्यक तथा मैत्रायणीय आरण्यक। सामवेदीय जैमिनी ब्राह्मण से सम्बद्ध तलवकार आरण्यक है। अथर्ववेद का कोई आरण्यक उपलब्ध नहीं है। इस प्रकार आज केवल आठ ही आरण्यक उपलब्ध हैं।

जब हम आरण्यकों में संगीत की चर्चा प्रारम्भ करते हैं तो संहिता के स्वरूप का ध्यान सर्वप्रथम करना आवश्यक हो जाता है। सामवेद की दो प्रकार की संहिता है। 1. आर्चिक संहिता तथा 2. गान संहिता। आर्चिक संहिता में जो ऋचाओं का संकलन है वह आग्नेय, इन्द्र पवमान तथा आरण्य काण्ड के अन्तर्गत आता है इसे पूर्वार्थिक तथा जिसमें तीन-तीन ऋचाओं का समूह है उसे उत्तरार्चिक कहते हैं। इस प्रकार आर्चिक संहिता के पूर्वार्चिक तथा उत्तरार्चिक दो रूप हैं। गान संहिता में इन्हीं ऋचाओं पर

1 तैत्ति० आ 2.20

2 वही, 2.22

3 शाखा० आ० 6.1

4 मै० आ० 6.9 
किये गये गान चार भागों में स्वरलिपि सहित संकलित है। जो इस प्रकार है - 1. ग्रामेगेय गान 2. आरण्यगेय गान 3 . ऊह गान तथा 4 . ऊह्यगान।

ग्रामेगेय गान आम समाज में तथा अध्ययन-अध्यापन में प्रयुक्त होते थे तथा ऊहगान का प्रयोग यज्ञा में किया जाता था। आरण्य जैसा कि नाम से विदित है इन गानों का अभ्यास मुनिजन वनों में करते थे। इन में बहुत विलक्षण स्तोभों का भी प्रयोग होता था। आरण्य के अनुरूप ऊह्य का प्रयोग भी वनों में मुनिजन करते थे इन्हें रहस्य गान भी कहा जाता है। जिन ऋषियों ने इनका दर्शन किया था उन्हें तो इन गानों की तकनीक, अर्थ आदि सब ज्ञान था लेकिन जिन्होंने इनका स्वयं दर्शन नहीं किया था उन्हें उपदेश के द्वारा ज्ञानवान बनाने की परम्परा चली। यज्ञ संस्था के विकास के साथ संगीत विशेषतया सामगान का प्रयोग अत्यधिक बढ़ गया और अधिक जटिल हो गया। इसी परिवेश में ब्राह्मण ग्रन्थों का सृजन हुआ। सामवेद के आठ ब्राह्मण जो आज भी उपलब्ध हैं संगीत की विशद व्याख्या करते हैं। किस यज्ञ में किस ऋक् पर किस साम का गान किस प्रकार किया जाएगा, इसकी पूर्ण पद्धति ब्राह्मणों में विशेष रूप से दी गयी है। वहाँ सामों का नाम, उनके निर्वचन, सामों की प्रशंसा उनके गान के फल, तकनीक में त्रुटि होने पर हानि आदि अर्थवाद का वर्णन भी किया गया है। विभिन्न वाद्ययन्त्रों वीणा, दुन्दुभि आदि का प्रयोग तथा नृत्य का प्रयोग भी उल्लिखित है। वस्तुतः सामवेद के ब्राह्मण ही सामविषयक पूर्ण जानकारी प्रदान करते हैं। इनमें सर्वत्र प्रवृत्ति मूलकता है।

आरण्यक ब्राह्मणों के परिशिष्ट माने जाते हैं किन्तु इनमें प्रवृत्तिमूलकता की अपेक्षा निवृत्युन्मुखता की प्रवृत्ति विशेष रूप से दृष्टिगोचर होती है। यही बात आरण्यकों में उल्लिखित सामों के विषय में भी ह। ब्राह्मणों में प्रयुक्त साम जहाँ कर्त्तव्य कर्मकाण्ड से सम्बद्ध है वहां आरण्यकों में इनके आध्यात्मिक एवं प्रतीकात्मक महत्त्व का विशेष प्रतिपादन हुआ है। ऐतरेय आरण्यक 1.3 .1 हिंकार का उल्लेख करते हुए उसका तादात्म्य ब्रह्म से मानता है। ${ }^{2}$ यहीं पर हिंकार को वृषा और ऋक् को योषा कहा गया है। दोनों के संयोग को मिथुन की संज्ञा दी गयी है जिसकी प्रशंसा में कहा है कि जो इस तथ्य को जानता है वह प्रजा तथा पशुओं से समृद्ध होता है। इसी प्रकार ऐ. आ. 1.2 .4 में एकविंश स्तोम की प्रशंसा की गयी है। ऐतरेय आú 2.3.4 में साम की प्रत्येक भक्ति की पाँच बार दोहरा कर गाने का उल्लेख है। इस प्रकार गान करने से हजार प्रकार के स्तोम बन जाते हैं। तैतिरीय आरण्यक में सामगान को ब्रह्मयज्ञ की संज्ञा दी गयी है तथा सामगान के स्वाध्याय को सोमरस की आहुति माना है। इस प्रकार सामगान जो कि तत्कालीन शास्त्रीय संगीत के रूप में प्रचलित था उसकी आध्यात्मिक व्याख्या आरण्यकों में प्राप्त होती है। मनुष्य शरीर को दैवी वीणा कहा है। नाद को जो प्राण तथा अग्नि का संयोग संगीतशास्त्र ग्रन्थों में कहा गया है उस प्राण की आदि भौतिक से लेकर आध्यात्मिक व्याख्या आरण्यकों में विशद

1 प्र० लेखिका द्वारा लिखित पुस्तक सामगान: उद्भव, व्यवहार एवं सिद्धान्त पृ० 55 तथा आगे

2 ब्रह्म वै हिंकारो। ऐ० आ० 1.3 .1

3 पञ्चकृत: प्रस्तौति, पज्चकृतव: उद्गायति, पझ्चकृतव: प्रतिहरति, पझ्चकृत्व: उपद्रवति, पञ्वकृत्वो निधनमुपयन्ति तत्स्तोम सहस्रं भवति। ऐ० आ० 2.3 .4

4 तैति० आ० 2.10 
रूप में प्राप्त होती है नाद ब्रह्म की कल्पना का मूल आरण्यक ही हैं। प्राण इस विश्व का धारक है प्राण की शक्ति से जैसे यह आकाश अपने स्थान पर स्थित है, इसी प्रकार सर्वोच्च प्राणी से लेकर चींटी तक समस्त प्राणी इसी प्राण के द्वारा प्रतिष्ठित हैं। प्राण ही आयु का कारण है। सभो ऋचायें यहाँ तक कि सभी वेद और ध्वनियां प्राण में ही सन्निहित हैं। ${ }^{2}$ प्राण के विभिन्न रूपों के ध्यान से ध्याता को विभिन्न फलों की प्राप्ति होती है। मैत्रायणी आरण्यक में प्राण अग्नि और परमात्मा को समानार्थक बतलाया गया है।

वाद्यों में दुन्दुभी तथा वीणा का उल्लेख प्राप्त होता है। तैतिरीय आरण्यक में भूमिदुन्दुभी का उल्लेख है। ${ }^{4}$ ऐतरेय आरण्यक में शरीर को दैवी वीणा माना है तथा इस दैवी वीणा के अनुरूप ही मनुष्य के द्वारा निर्मित वीणा को काष्ठ वीणा कहा है। इसके वर्णन में वीणा के सभी अंगों का उल्लेख है जिससे यह ज्ञात होता है कि उस समय वीणा का निर्माण किस प्रकार किया जाता था। ${ }^{5}$ ठाकुर जयदेव सिंह ने रेखाचित्रों सहित किन्हीं वीणाओं का उल्लेख किया है। उनके अनुसार वीणा प्राय: धनुषाकार होती थी तथा कुछ भी वीणायें थीं जो धनुषाकार न होकर सीधे काठ से निर्मित हाती थी। धनुषाकार वीणायें जिन्हें पहले 'वाण' कहते थे, भारत में बहुत समय तक प्रचलित रहीं। सीधी वीणा में दण्ड कभी-कभी बांस का होता था। जिससे वीणा बजायी जाती थी उसे वादन या कोण कहते हैं। नृत्य का उल्लेख आरण्यकों में अभी शोध का विषय है।

उपर्युक्त समीक्षा के आधार पर निष्कर्ष रूप में इस प्रकार व्यक्त किया जा सकता है-

यज्ञ संस्था के आध्यात्मिक और प्रतीकात्मक पक्षों की अभिव्यक्ति की दिशा में आरण्यक साहित्य महीयसी भूमिका का निर्वाहक है। उपनिषदों के तात्तिक अनुशीलन के सन्दर्भ में आरण्यक साहित्य पूर्वपीठिका का निर्माता है। अनके नये और ऐतिहासिक, सांगीतिक एवं सांस्कृतिक, तथ्यों की प्रस्तुति के कारण यह साहित्य विशेष उपादेय है। ब्राह्मण और उपनिषदों के मध्य की भाषा और शैली के विकास क्रम का इससे परिज्ञान होता है। नैतिकता और आचार-दर्शन की दृष्टि से आरण्यक साहित्य मानवीय मानस के उर्ध्वारोहण में परम सहायक है।

अतः अन्य ग्रन्थों की भान्ति आरण्यग्रन्थों का भी अध्ययन होना चाहिये। इस दिशा में शोध करने से कई नवीन तथ्य उद्घाटित होंगे तथा संगीत के इतिहास तथा दर्शन की परम्परा सुदृढ़ होगी।

1 ऐत० आ० 2.1.6

2 ता वा ऐता सर्वा ऋचा: घोषा एकैव व्याहित:।

प्राण एव प्राण ऋक् इत्येव विद्यात्। ऐ० आ० 2.2.2

3 प्राणो अग्नि: परमात्मा। मै० आ० 6.9

4 तैति० आ० 5.1 .5

5 ऐत० आ० 3.5.11

6 द्र० भारतीय संगीत का इतिहास, पृ० 79

7 वही पृ० 80 